\title{
Rosai Dorfman Disease Presenting as Isolated Cervical Lymphadenopathy
}

\author{
Pratik Savant ${ }^{1}$, Dattaprasad Samant ${ }^{2}$, Anant Ramani ${ }^{3}$, F.P. Noronha ${ }^{4}$ \\ ${ }^{1}$ Department of Surgery, Goa Medical College, Goa, India. ${ }^{2}$ Department of Surgery, Goa \\ Medical College, Goa, India. ${ }^{3}$ Department of Surgery, Goa Medical College, Goa, India. \\ ${ }^{4}$ Department of Surgery, Goa Medical College, Goa, India.
}

\section{PRESENTATION OF CASE}

A 40-year-old male presented in our surgery OPD with a history of left sided neck swelling since two months. There was no associated history of pain, fever or cough. General physical and systemic examination did not reveal any abnormality. Local examination revealed multiple cervical lymph nodes along the jugular chain, largest measuring $3 \mathrm{cms}$. His complete blood count and chest X-ray did not reveal any abnormality. FNAC was suggestive of sinus histiocytosis. Patient underwent excision of the lymph nodes under general anaesthesia. Since the submandibular gland also appeared involved on table, decision was taken to excise it as well. Histopathology report revealed multiple foci of dilatation of sinusoids with histiocytes showing engulfment of lymphocytes and plasma cells, thus consistent with Rosai Dorfman disease. The Submandibular Gland was normal. The Patient was managed conservatively. The postoperative recovery was uneventful and subsequent follow ups did not reveal any recurrence.

Isolated painless cervical lymphadenopathy is a common presentation in a surgical OPD accounting for almost $12-15 \%$ of all cases ${ }^{1}$. Most common differentials in these cases include infections, tuberculosis, lymphoma, and metastatic malignancies. We report a case of Rosai Dorfman disease presenting as isolated unilateral cervical lymphadenopathy in a young male. The diagnosis was made on cytology and later confirmed on histopathology of excision biopsy. The patient was managed conservatively and had an uneventful course.

Rosai Dorfman disease (sinus histiocytosis with massive lymphadenopathy) is a rare benign proliferative disorder of histiocytes, characterised in majority of cases by painless ipsilateral cervical lymph node enlargement and rarely as an extranodal disease $^{2,3}$. In the setting of other conspicuous differentials, it often presents as a histopathological surprise. This disease often has a benign course and mostly resolves spontaneously. Our case emphasizes on the need for unbiased general approach in the management of cervical lymphadenopathy with the awareness of Rosai Dorfman as a differential diagnosis.
Corresponding Author: Dr. Pratik Savant, Junior Resident, Department of Surgery, Goa Medical College, Goa, India.

E-mail: pratiksavant@gmail.com

DOI: $10.14260 / j e m d s / 2020 / 621$

How to Cite This Article:

Savant P, Samant D, Ramani A, et al. Rosai Dorfman disease presenting as isolated cervical lymphadenopathy. J Evolution Med Dent Sci 2020;9(38):2850-2851, DOI: $10.14260 / \mathrm{jemds} / 2020 / 621$

Submission 20-12-2018,

Peer Review 04-08-2020,

Acceptance 10-08-2020,

Published 21-09-2020.

Copyright (c) 2020 JEMDS. This is an open access article distributed under Creative Commons Attribution License [Attribution 4.0 International (CC BY 4.0)] 


\section{DIFFERENTIAL DIAGNOSIS}

Tuberculosis, lymphoma and metastatic malignancies. ${ }^{1}$
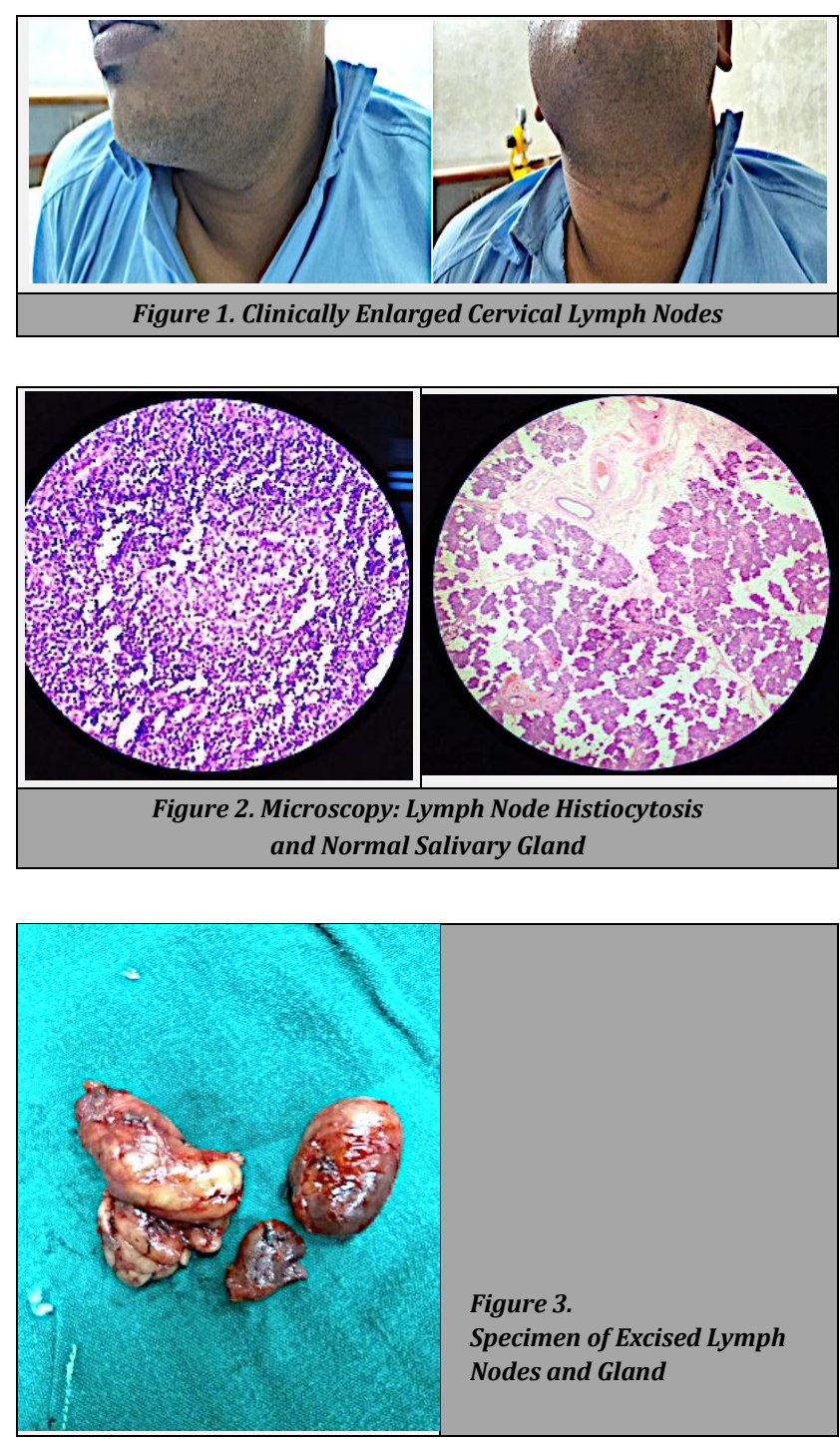

Figure 3.

Specimen of Excised Lymph Nodes and Gland

\section{DISCUSSION}

No definitive treatment protocols exist in view of uncertain aetiology and rare occurrence 4 . Most symptomatic cases resolve over time without any treatment ${ }^{4}$. Symptomatic treatment may be required in a few cases.

Rarely, extensive type of Rosai Dorfman may present with generalised painful lymphadenopathy which would require immunosuppressive or systemic chemotherapy5.

\section{CONCLUSIONS}

Rosai Dorfman Disease is a rare, benign cause of cervical lymphadenopathy. Biopsy is diagnostic in most of the cases. Majority of the cases resolve spontaneously and require just regular follow up. Symptomatic treatment may be required in a few cases, especially with extranodal involvement ${ }^{6}$.

Financial or Other Competing Interests: None.

\section{REFERENCES}

[1] Allhiser JN, Mcknight TA, Shank JC. Lymphadenopathy in a family practice. J Fam Pract 1981;12(1):27-32.

[2] Tiong T, Subramaniam S, Swethadhri G. Rosai-Dorfman disease: a case report. The Internet Journal of Otorhinolaryngology 2006;6(1):1-5.

[3] Rosa J, Dorfman RF. Sinus histiocytosis with massive lymphadenopathy. A newly recognized benign clinicopathological entity. Arch Pathol 1969;87(1):63-70.

[4] Levine PH, Jahan N, Murari P, et al. Detection of human herpesvirus 6 in tissues involved by sinus histiocytosis with massive lymphadenopathy (Rosai-Dorfman disease). J Infect Dis 1992;166(2):291-5.

[5] Lipton JM. The pathogenesis, diagnosis, and treatment of histiocytosis syndromes. Pediatric Dermatology 1983;1(2):112-20.

[6] Komp DM. The treatment of sinus histiocytosiswith massive lymphadenopathy (Rosai-Dorfman Disease). Semin Diagn Pathol 1990;7(1):83-6. 